\title{
On some tractable and hard instances for partial incentives and target set selection
}

\author{
Stefan Ehard Dieter Rautenbach \\ Institut für Optimierung und Operations Research, Universität Ulm, Ulm, Germany, \\ \{stefan.ehard, dieter.rautenbach\}@uni-ulm.de
}

\begin{abstract}
A widely studied model for influence diffusion in social networks are target sets. For a graph $G$ and an integer-valued threshold function $\tau$ on its vertex set, a target set or dynamic monopoly is a set of vertices of $G$ such that iteratively adding to it vertices $u$ of $G$ that have at least $\tau(u)$ neighbors in it eventually yields the entire vertex set of $G$. This notion is limited to the binary choice of including a vertex in the target set or not, and Cordasco et al. proposed partial incentives as a variant allowing for intermediate choices.

We show that finding optimal partial incentives is hard for chordal graphs and planar graphs but tractable for graphs of bounded treewidth and for interval graphs with bounded thresholds. We also contribute some new results about target set seletion on planar graphs by showing the hardness of this problem, and by describing an efficient $O(\sqrt{n})$-approximation algorithm as well as a PTAS for the dual problem of finding a maximum degenerate set.
\end{abstract}

Keywords: Dynamic monopoly; target set; partial incentive; degenerate set; chordal graph; planar graph; treewidth; interval graph

\section{Introduction}

Target sets, also known as dynamic monopolies, are a popular model for social influence and the spread of opinions in social networks [16,19]. For unrestricted instances, optimal target sets are hard to find [11,12], and efficient algorithms are only known for quite restricted instances such as treestructured graphs [7, 8, 21]. Constructing a target set involves binary decisions for every vertex of the underlying graph, and Cordasco et al. 14 recently proposed so-called partial incentives as a more subtle way of influencing a network. Next to hardness results and bounds similar to those known for target sets [2,12,23], they describe an efficient algorithm computing optimal partial incentives for trees and complete graphs. In the present paper we present several new results concerning the complexity as well as tractable cases of partial incentives and (the dual of) target sets in planar graphs.

Before we can explain our contribution in detail, we need some terminology and notation.

We only consider finite, simple, and undirected graphs. A threshold function for a graph $G$ is a function from its vertex set $V(G)$ to the set of integers. Let $\tau \in \mathbb{Z}^{V(G)}$ be a threshold function for $G$. For a set $D$ of vertices of $G$, the hull $H_{(G, \tau)}(D)$ of $D$ in $(G, \tau)$ is the smallest set $H$ of vertices of $G$ such that $D \subseteq H$, and $u \in H$ for every vertex $u$ of $G$ with $\left|H \cap N_{G}(u)\right| \geq \tau(u)$. The set $H_{(G, \tau)}(D)$ is obtained by starting with $D$, and iteratively adding vertices $u$ that have at least $\tau(u)$ neighbors in the 
current set as long as possible. A set $D$ of vertices of $G$ is a dynamic monopoly or a target set of $(G, \tau)$ if $H_{(G, \tau)}(D)=V(G)$. The minimum order of a dynamic monopoly of $(G, \tau)$ is denoted by $\operatorname{dyn}(G, \tau)$.

It has been observed several times [2,12,23] that the dual of the notion of a dynamic monopoly is the notion of a degenerate set. For a function $\kappa \in \mathbb{Z}^{V(G)}$, a set $I$ of vertices of $G$ is $\kappa$-degenerate in $G$ if there is a linear order $u_{1}, \ldots, u_{k}$ of the vertices in $I$ such that $u_{i}$ has at most $\kappa\left(u_{i}\right)$ neighbors in $\left\{u_{j}: j \in[i-1]\right\}$ for every $i \in[k]$, where $[n]$ denotes the set of positive integers at most $n$ for every integer $n$, and $[n]_{0}=[n] \cup\{0\}$. The maximum cardinality of a $\kappa$-degenerate set of $G$ is denoted by $\alpha(G, \kappa)$. If $\kappa, \tau \in \mathbb{Z}^{V(G)}$ are two functions such that the degree $d_{G}(u)$ of every vertex $u$ of $G$ equals $\tau(u)+\kappa(u)$, then a set $I$ of vertices of $G$ is $\kappa$-degenerate in $G$ if and only if $V(G) \backslash I$ is a dynamic monopoly of $(G, \tau)$. This duality generalizes the well-known duality between independent sets and vertex covers.

A function $\sigma \in \mathbb{N}_{0}^{V(G)}$ is a partial incentive of $(G, \tau)$ [14] if $H_{(G, \tau-\sigma)}(\emptyset)=V(G)$, that is, reducing the initial thresholds $\tau$ as specified by $\sigma$, the empty set becomes a dynamic monopoly. Throughout this paper, we define the weight of a function $f$ with domain $D$ as $f(D)=\sum_{d \in D} f(d)$. Let $\operatorname{pi}(G, \tau)$ denote the minimum weight of a partial incentive of $(G, \tau)$, and a partial incentive of $(G, \tau)$ of weight $\operatorname{pi}(G, \tau)$ is called optimal.

Our first contributions are complexity results.

We show NP-completeness for dynamic monopolies in planar graphs, which appears to be unknown.

Theorem 1.1. For a given triple $(G, \tau, k)$, where $G$ is a planar graph, $\tau$ is a threshold function for $G$, and $k$ is a positive integer, it is $N P$-complete to decide whether $\operatorname{dyn}(G, \tau) \leq k$.

Exploiting results from [7,8], we establish the following hardness results for partial incentives.

Theorem 1.2. For a given pair $(G, \tau)$, where $G$ is a graph of order $n$ and treewidth $w$, and $\tau$ is a threshold function for $G$, an optimal partial incentive of $(G, \tau)$ cannot be computed in time $n^{o(\sqrt{w})}$ unless all problems in SNP can be solved in sub-exponential time.

Theorem 1.3. For a given triple $(G, \tau, k)$, where $G$ is a chordal graph, $\tau$ is a threshold function for $G$, and $k$ is a positive integer, it is $N P$-complete to decide whether $\operatorname{pi}(G, \tau) \leq k$.

Finally, using Theorem 1.1, we obtain the following.

Corollary 1.4. For a given triple $(G, \tau, k)$, where $G$ is a planar graph, $\tau$ is a threshold function for $G$, and $k$ is a positive integer, it is $N P$-complete to decide whether $\operatorname{pi}(G, \tau) \leq k$.

Our remaining contributions are algorithmic. We first show two tractable cases for partial incentives.

Theorem 1.5. Let $w$ be a non-negative integer.

For a given pair $(G, \tau)$, where $G$ is a graph of order $n$ and treewidth at most $w$, and $\tau$ is a threshold function for $G$, an optimal partial incentive of $(G, \tau)$ can be computed in time $n^{O(w)}$.

Theorem 1.6. Let $t$ be a non-negative integer.

For a given pair $(G, \tau)$, where $G$ is an interval graph of order $n$, and $\tau$ is a threshold function for $G$ with $\tau(u) \leq t$ for every vertex $u$ of $G$, an optimal partial incentive of $(G, \tau)$ can be determined in time $n^{O\left(t^{2}\right)}$. 
Complementing our hardness result for dynamic monopolies in planar graphs, we contribute approximation algorithms. We give a PTAS for degenerate sets in planar graph using Baker's layering technique [5].

Theorem 1.7. Let $\epsilon$ be a positive real number.

For a given pair $(G, \kappa)$, where $G$ is a planar graph and $\kappa \in \mathbb{Z}^{V(G)}$, a $\kappa$-degenerate set $I$ in $G$ with $|I| \geq(1-\epsilon) \alpha(G, \kappa)$ can be determined in polynomial time.

The main techniques for constructing PTASs on planar graphs such as Baker's layering technique [5] and the bidimensionality theory [15] seem not to work for finding a minimum dynamic monopoly, the dual problem of a maximum degenerate set. On the positive side, we show the following.

Theorem 1.8. For a given pair $(G, \tau)$, where $G$ is a graph of order $n$ and size $m$, and $\tau$ is a threshold function for $G$, and for a given tree-decomposition of $G$ of width $w$ and size $O(n)$, a dynamic monopoly $D$ of $(G, \tau)$ with $|D| \leq(w+1) \operatorname{dyn}(G, \tau)$ can be determined in time $O\left(n^{2} m\right)$.

Corollary 1.9. For a given pair $(G, \tau)$, where $G$ is a planar graph of order $n$, and $\tau$ is a threshold function for $G$, a dynamic monopoly $D$ of $(G, \tau)$ with $|D| \leq O(\sqrt{n}) \operatorname{dyn}(G, \tau)$ can be determined in time $O\left(n^{3}\right)$.

All proofs are given in the next section.

\section{Proofs}

Our hardness results follow from known results using two simple constructions described in Lemma 2.1 and Lemma 2.2 below.

Lemma 2.1. Let $G$ be a graph of order n. Let $G^{\prime}$ arise from $G$ by adding, for every edge uv of $G$, an independent set $I(u v)$ of order $n$ as well as all $2 n$ possible edges between $\{u, v\}$ and $I(u v)$. Let

$$
\tau^{\prime}: V\left(G^{\prime}\right) \rightarrow \mathbb{Z}: u \mapsto \begin{cases}d_{G}(u) n & , \text { if } u \in V(G), \text { and } \\ 1 & , \text { otherwise. }\end{cases}
$$

(i) The minimum order of a vertex cover of $G$ equals $\operatorname{dyn}\left(G^{\prime}, \tau^{\prime}\right)$.

(ii) If $G$ is planar, then $G^{\prime}$ is planar.

Proof. (i) First, let $X$ be a vertex cover of $G$. Let $H$ be the hull of $X$ in $\left(G^{\prime}, \tau^{\prime}\right)$. Since every vertex in $V\left(G^{\prime}\right) \backslash V(G)$ has a neighbor in $X$, and threshold value 1 , the set $H$ contains $V\left(G^{\prime}\right) \backslash V(G)$. Therefore, for every vertex $u$ of $G^{\prime}$ in $V(G) \backslash X$, the set $H$ contains all $d_{G}(u) n$ neighbors of $u$ in $V\left(G^{\prime}\right) \backslash V(G)$, which implies that $X$ is a dynamic monopoly of $\left(G^{\prime}, \tau^{\prime}\right)$.

Next, let $D$ be a dynamic monopoly of $\left(G^{\prime}, \tau^{\prime}\right)$. Since replacing a vertex in $D \backslash V(G)$ by some neighbor in $V(G)$ yields a dynamic monopoly, we may assume that $D \subseteq V(G)$. Suppose, for a contradiction, that $u, v \notin D$ for some edge $u v$ in $G$. Since $\left|N_{G^{\prime}}(u) \backslash(\{v\} \cup I(u v))\right| \leq\left(d_{G}(u)-1\right)(n+1)<$ $d_{G}(u) n$ and $\left|N_{G^{\prime}}(v) \backslash(\{u\} \cup I(u v))\right| \leq\left(d_{G}(v)-1\right)(n+1)<d_{G}(v) n$, we obtain a contradiction to the choice of $D$. Hence, $D$ is a vertex cover of $G$.

This completes the proof of (i).

(ii) This is obvious. 
Our first hardness result states the NP-completeness of dynamic monopolies for planar graphs, which quite surprisingly seems to be unknown.

Proof of Theorem 1.1. The stated problem is clearly in NP, and NP-completeness follows from the NP-completeness of VERTEx Cover for planar graphs [18] using Lemma 2.1.

For our remaining hardness results, we use the following lemma relating dynamic monopolies with partial incentives.

Lemma 2.2. Let $G$ be a graph of order $n$, and let $\tau$ be a threshold function for $G$. Let $G^{\prime}$ arise from $G$ by adding, for every vertex $u$ of $G$ with $\tau(u)>0$, a path $P_{u}$ of order $\tau(u)$ as well as all $\tau(u)$ possible edges between $u$ and the vertices of $P_{u}$. Let

$$
\tau^{\prime}: V\left(G^{\prime}\right) \rightarrow \mathbb{Z}: u \mapsto \begin{cases}\tau(u) & , \text { if } u \in V(G), \text { and } \\ 1 & , \text { otherwise. }\end{cases}
$$

(i) $\operatorname{dyn}(G, \tau)=\operatorname{pi}\left(G^{\prime}, \tau^{\prime}\right)$.

(ii) If $G$ has treewidth $w$, then $G^{\prime}$ has treewidth at most $\max \{w, 2\}$.

(iii) If $G$ is planar, then $G^{\prime}$ is planar.

(iv) If $G$ is chordal, then $G^{\prime}$ is chordal.

Proof. (i) First, let $D$ be a dynamic monopoly for $(G, \tau)$, where we may assume that $\tau(u)>0$ for every vertex $u$ in $D$. For every vertex $u$ in $D$, let $p_{u}$ be an endvertex of $P_{u}$. Since

$$
\sigma: V\left(G^{\prime}\right) \rightarrow \mathbb{N}_{0}: v \mapsto \begin{cases}1 & , \text { if } v=p_{u} \text { for some } u \in D, \text { and } \\ 0 & , \text { otherwise }\end{cases}
$$

is a partial incentive of $\left(G^{\prime}, \tau^{\prime}\right)$, and $\sigma\left(V\left(G^{\prime}\right)\right)=|D|$, we obtain $\operatorname{dyn}(G, \tau) \geq \operatorname{pi}\left(G^{\prime}, \tau^{\prime}\right)$.

Next, let $\sigma$ be a partial incentive of $\left(G^{\prime}, \tau^{\prime}\right)$. In view of the definition of $\tau^{\prime}$, we may assume that $\sigma(V(G))=0$, and $\sigma\left(V\left(P_{u}\right)\right) \leq 1$ for every vertex $u$ in $V(G)$. Since $D=\left\{u \in V(G): \sigma\left(V\left(P_{u}\right)\right)=1\right\}$ is a dynamic monopoly for $(G, \tau)$, and $|D|=\sigma\left(V\left(G^{\prime}\right)\right)$, we obtain $\operatorname{dyn}(G, \tau) \leq \operatorname{pi}\left(G^{\prime}, \tau^{\prime}\right)$.

(ii) Let $\left(T,\left(X_{t}\right)_{t \in V(T)}\right)$ be a tree-decomposition of $G$, cf. the beginning of Subsection 2.1 Let $u$ be a vertex of $G$ with $\tau(u)=k>0$. Let $P_{u}=v_{1} \ldots v_{k}$. Let $t$ be a vertex of $T$ with $u \in X_{t}$. Attaching at $t$ within $T$ a path $t_{1} \ldots t_{k}$, and setting $X_{t_{1}}=\left\{u, v_{1}\right\}, X_{t_{2}}=\left\{u, v_{1}, v_{2}\right\}, \ldots, X_{t_{k}}=\left\{u, v_{k-1}, v_{k}\right\}$ yields a tree-decomposition of $G \cup P_{u}$ of width at $\operatorname{most} \max \{w, 2\}$. Proceeding similarly for all other vertices of $G$ with positive threshold value yields the desired statement, because $\max \{\max \{w, 2\}, 2\}=\max \{w, 2\}$.

(iii) and (iv) are obvious.

It is now easy to show the hardness of partial incentives exploiting results from [7, 8 .

Proof of Theorem 1.2. This follows easily from Theorem 1.2 in [7. In fact, in order to compute an optimal dynamic monopoly for a given pair $(G, \tau)$, where $G$ is a graph of order $n$, and $\tau$ is a threshold function for $G$, a simple algorithmic reduction allows to assume that $\tau(u)<n$ for every vertex $u$ of $G$. This implies that the graph $G^{\prime}$ constructed in Lemma 2.2 has order $n^{\prime}$ at most $n^{2}$, and an algorithm 
computing an optimal partial incentive of $\left(G^{\prime}, \tau^{\prime}\right)$ as in Lemma 2.2 in time $\left(n^{\prime}\right)^{o(\sqrt{w})}$, would allow to compute an optimal dynamic monopoly of $(G, \tau)$ in time $n^{2 o(\sqrt{w})}=n^{o(\sqrt{w})}$.

Proof of Theorem 1.3. The stated problem is clearly in NP, and NP-completeness follows from Theorem 1.3 in [8] using Lemma 2.2 as in the previous proof.

Corollary 1.4 follows easily from Theorem 1.1 and Lemma 2.2 .

We proceed to the two tractable cases.

\subsection{Partial incentives of graphs of bounded treewidth}

We need the notion of a nice tree-decomposition introduced by Kloks [20]. For a graph $G$, a treedecomposition of $G$ is a pair $\left(T,\left(X_{t}\right)_{t \in V(T)}\right)$, where $T$ is a tree and $\left(X_{t}\right)_{t \in V(T)}$ is a collection of sets of vertices of $G$ satisfying the following properties:

- $\bigcup_{t \in V(T)} X_{t}=V(G)$,

- for every edge $u v$ of $G$, there is a set $X_{t}$ containing both $u$ and $v$, and

- for every vertex $u$ of $G$, the set $\left\{t \in V(T): u \in X_{t}\right\}$ induces a subtree of $T$.

The width of the tree-decomposition is $\max _{t \in V(T)}\left|X_{t}\right|-1$, and the treewidth of $G$ is the minimum width of a tree-decomposition of $G$. For a rooted tree-decomposition $\left(T,\left(X_{t}\right)_{t \in V(T)}\right)$ and for every node $t$ of $T$, let $V_{t}$ denote the set of nodes of $T$ that contains $t$ and all its descendants, and, let $G_{t}$ be the subgraph of $G$ induced by $\bigcup_{s \in V_{t}} X_{s}$. A tree-decomposition $\left(T,\left(X_{t}\right)_{t \in V(T)}\right)$ of $G$ is nice if $T$ is a rooted binary tree, and every node $t$ of $T$ is of one of the following types:

- $t$ is a leaf of $T$, and $X_{t}=\emptyset$ (leaf node).

- $t$ has two children $t^{\prime}$ and $t^{\prime \prime}$, and $X_{t}=X_{t^{\prime}}=X_{t^{\prime \prime}}($ join node) .

- $t$ has a unique child $t^{\prime}$, and

either $\left|X_{t} \backslash X_{t^{\prime}}\right|=1$ and $\left|X_{t^{\prime}} \backslash X_{t}\right|=0$ (introduce node), or $\left|X_{t^{\prime}} \backslash X_{t}\right|=1$ and $\left|X_{t} \backslash X_{t^{\prime}}\right|=0$ (forget node).

For a linear order $\prec$ on a set $X$, and two subsets $X_{1}$ and $X_{2}$ of $X$, we write $X_{1} \prec X_{2}$ if $x_{1} \prec x_{2}$ for every $x_{1} \in X_{1}$ and $x_{2} \in X_{2}$.

Proof of Theorem 1.5. Let $(G, \tau)$ be as in the statement of the theorem.

In view of the desired statement, we may assume that $G$ has treewidth exactly $w$.

We may assume that $\tau(u)<n$ for every vertex $u$ of $G$; otherwise, we compute an optimal partial incentive $\sigma^{\prime}$ of $\left(G, \tau^{\prime}\right)$, where

$$
\tau^{\prime}(u)=\tau(u)-\max \{\tau(u)-(n-1), 0\}<n \text { for every vertex } u \text { of } G,
$$

and return the partial incentive $\sigma$ of $(G, \tau)$ with $\sigma(u)=\sigma^{\prime}(u)+\max \{\tau(u)-(n-1), 0\}$ for every vertex $u$ of $G$. It is easy to see that $\sigma$ is optimal.

In time $n^{O(w)}$ [4,20] we can determine a nice tree-decomposition $\left(T,\left(X_{t}\right)_{t \in V(T)}\right)$ of $G$ of width at most $w$ such that $n(T)=O(w n)$. Let $r$ be the root of $T$. 
Our approach is dynamic programming on the nice tree-decomposition and to propagate information in a bottom-up fashion within $T$. For every node $t$ in $T$, we consider

(i) all possible candidates $\sigma_{t}$ for the restriction of an optimal partial incentive $\sigma$ of $(G, \tau)$ to $X_{t}$,

(ii) all possible linear orders $\prec_{t}$ in which the elements of $X_{t}$ may enter the hull $H_{(G, \tau-\sigma)}(\emptyset)$, and

(iii) all possible amounts $\rho_{t}$ of help that each vertex in $X_{t}$ receives from outside of $G_{t}$ when it enters the hull.

Accordingly, we define the notion of a local cascade $\left(\sigma_{t}, \prec_{t}, \rho_{t}\right)$ for $G_{t}$, where

(i) $\sigma_{t}: X_{t} \rightarrow[n-1]_{0}$,

(ii) $\prec_{t}$ is a linear order on $X_{t}$ with $\left\{u \in X_{t}: \tau(u)-\sigma_{t}(u) \leq 0\right\} \prec_{t}\left\{v \in X_{t}: \tau(v)-\sigma_{t}(v)>0\right\}$, and

(iii) $\rho_{t}: X_{t} \rightarrow[n-1]_{0}$.

Since $\left|X_{t}\right| \leq w+1 \leq n$, there are $O\left((w+1) ! n^{O(w)}\right)=n^{O(w)}$ local cascades for $G_{t}$.

For a local cascade $\left(\sigma_{t}, \prec_{t}, \rho_{t}\right)$ for $G_{t}$, let $\pi_{t}\left(\sigma_{t}, \prec_{t}, \rho_{t}\right)$ be a function $\sigma \in[n-1]_{0}^{V\left(G_{t}\right)}$ of minimum cost such that $\left.\sigma\right|_{X_{t}}=\sigma_{t}$, and

(iv) there is a linear extension $\prec$ of $\prec_{t}$ to $V\left(G_{t}\right)$ such that, for every vertex $u$ of $G_{t}$,

- either $u \in V\left(G_{t}\right) \backslash X_{t}$ and $\left|N_{G_{t}}^{\prec}(u)\right| \geq \tau(u)-\sigma(u)$,

- or $u \in X_{t}$ and $\left|N_{G_{t}}^{\prec}(u)\right| \geq \tau(u)-\sigma(u)-\rho_{t}(u)$,

where $N_{G_{t}}^{\prec}(u)$ denotes the set of neighbors of $u$ in $G_{t}$ that appear before $u$ in the linear order $\prec$. If no such function $\sigma$ exists, we set all the values of $\pi_{t}\left(\sigma_{t}, \prec_{t}, \rho_{t}\right)$ to $\infty$. Note that $\pi_{t}\left(\sigma_{t}, \prec_{t}, \rho_{t}\right)$ is not necessarily unique.

Since $G_{r}=G$, and no vertex in $X_{r}$ has a neighbor outside of $V\left(G_{r}\right)$, the following claim is obvious from the definitions.

Claim 2.1. If $\left(\sigma_{r}, \prec_{r}, 0\right)$ is a local cascade for $G_{r}$ that minimizes the cost of $\pi_{r}\left(\sigma_{r}, \prec_{r}, 0\right)$, then $\pi_{r}\left(\sigma_{r}, \prec_{r}, 0\right)$ is an optimal partial incentive of $(G, \tau)$.

We now explain how to compute $\pi_{t}\left(\sigma_{t}, \prec_{t}, \rho_{t}\right)$ for each node $t$ of $T$ using dynamic programming.

Since $X_{t}=\emptyset$ for every leaf node $t, \pi_{t}\left(\sigma_{t}, \prec_{t}, \rho_{t}\right)$ is initialized as an empty function for such a $t$.

Claim 2.2. For an introduce or a forget node $t$ with child node $t^{\prime}$, and for every local cascade $\left(\sigma_{t}, \prec_{t}, \rho_{t}\right)$ for $G_{t}$, given $\pi_{t^{\prime}}\left(\sigma_{t^{\prime}}, \prec_{t^{\prime}}, \rho_{t^{\prime}}\right)$ for all local cascades $\left(\sigma_{t^{\prime}}, \prec_{t^{\prime}}, \rho_{t^{\prime}}\right)$ for $G_{t^{\prime}}, \pi_{t}\left(\sigma_{t}, \prec_{t}, \rho_{t}\right)$ can be computed in time $n^{O(w)}$.

Proof of Claim 2.2. First, we assume that $t$ is an introduce node, that is, $X_{t} \backslash X_{t^{\prime}}=\{u\}$ for some vertex $u$ of $G$ that does not belong to $G_{t^{\prime}}$. Clearly, $N_{G_{t}}(u) \subseteq X_{t^{\prime}}$. If $\left|N_{G\left[X_{t}\right]}^{\prec t}(u)\right|<\tau(u)-\sigma_{t}(u)-\rho_{t}(u)$, then no function satisfies condition (iv), and we set all the values of $\pi_{t}\left(\sigma_{t}, \prec_{t}, \rho_{t}\right)$ to $\infty$. Now, let $\left|N_{G\left[X_{t}\right]}^{\prec t}(u)\right| \geq \tau(u)-\sigma_{t}(u)-\rho_{t}(u)$. Every neighbor of $u$ in $X_{t^{\prime}}$ that appears after $u$ in the linear order $\prec_{t}$ receives one additional unit of help from $u$ when it enters the hull. Therefore, for every vertex $v \in X_{t^{\prime}}$, let

$$
\rho_{t^{\prime}}(v)= \begin{cases}\rho_{t}(v)+1 & , \text { if } u v \in E(G) \text { and } u \prec_{t} v, \text { and } \\ \rho_{t}(v) & , \text { otherwise. }\end{cases}
$$


Now, the function $\pi_{t}\left(\sigma_{t}, \prec_{t}, \rho_{t}\right)$ on $V\left(G_{t}\right)$ can be chosen as

$$
\pi_{t}\left(\sigma_{t}, \prec_{t}, \rho_{t}\right)(v)= \begin{cases}\sigma_{t}(u) & , \text { if } v=u, \text { and } \\ \pi_{t^{\prime}}\left(\sigma_{t^{\prime}}, \prec_{t^{\prime}}, \rho_{t^{\prime}}\right)(v) & , \text { otherwise, }\end{cases}
$$

where $\sigma_{t^{\prime}}$ and $\prec_{t^{\prime}}$ are the restrictions of $\sigma_{t}$ and $\prec_{t}$ to $X_{t^{\prime}}$, respectively. Clearly, the computation of $\pi_{t}\left(\sigma_{t}, \prec_{t}, \rho_{t}\right)$ can be done in time $n^{O(w)}$.

Next, we assume that $t$ is a forget node, that is, $X_{t^{\prime}} \backslash X_{t}=\{u\}$ for some vertex $u$ of $G_{t}$, and $G_{t}=G_{t^{\prime}}$. For every $i \in[n-1]_{0}$, let $\sigma_{i}$ be the function on $X_{t^{\prime}}$ defined as

$$
\sigma_{i}(x)= \begin{cases}i & , \text { if } x=u, \text { and } \\ \sigma_{t}(x) & , \text { otherwise }\end{cases}
$$

For every $j \in\left|X_{t^{\prime}}\right|$, let $\prec_{j}$ be a linear extension of $\prec_{t}$ to $X_{t^{\prime}}$ such that the vertex $u$ is the $j$ th vertex in the order $\prec_{j}$. Note that $v$ does not have any neighbors outside of $V\left(G_{t}\right)$. Therefore, for every vertex $v \in X_{t^{\prime}}$, let

$$
\rho_{t^{\prime}}(v)= \begin{cases}0 & , \text { if } v=u, \text { and } \\ \rho_{t}(v) & , \text { otherwise. }\end{cases}
$$

Now, the function $\pi_{t}\left(\sigma_{t}, \prec_{t}, \rho_{t}\right)$ can be chosen as the function $\pi_{t^{\prime}}\left(\sigma_{i}, \prec_{j}, \rho_{t^{\prime}}\right)$ that minimizes the cost among all choices of $i \in[n-1]_{0}$ and $j \in\left|X_{t^{\prime}}\right|$. Clearly, this can also be done in time $n^{O(w)}$.

Claim 2.3. For every join node $t$ with children nodes $t^{\prime}$ and $t^{\prime \prime}$, and for every local cascade $\left(\sigma_{t}, \prec_{t}, \rho_{t}\right)$ for $G_{t}$, given $\pi_{s}\left(\sigma_{s}, \prec_{s}, \rho_{s}\right)$ for all local cascades $\left(\sigma_{s}, \prec_{s}, \rho_{s}\right)$ for $G_{s}$ and both $s \in\left\{t^{\prime}, t^{\prime \prime}\right\}, \pi_{t}\left(\sigma_{t}, \prec_{t}, \rho_{t}\right)$ can be computed in time $n^{O(w)}$.

Proof of Claim [2.3. By the definition of a nice tree decomposition, $G_{t}=G_{t^{\prime}} \cup G_{t^{\prime \prime}}$ and $X_{t}=X_{t^{\prime}}=$ $X_{t^{\prime \prime}}=V\left(G_{t^{\prime}}\right) \cap V\left(G_{t^{\prime \prime}}\right)$, in particular, there are no edges between $V\left(G_{t^{\prime}}\right) \backslash X_{t}$ and $V\left(G_{t^{\prime \prime}}\right) \backslash X_{t}$.

Let $\rho_{\text {int }} \in[n-1]_{0}^{X_{t}}$ be such that

$$
\rho_{\text {int }}(u)=\max \left\{\tau(u)-\sigma_{t}(u)-\rho_{t}(u)-\left|N_{G\left[X_{t}\right]}^{\prec t}(u)\right|, 0\right\}
$$

for every vertex $u$ in $X_{t}$, that is, $\rho_{\text {int }}(u)$ is the (minimum) number of neighbors that $u$ needs in $V\left(G_{t}\right) \backslash X_{t}$ when entering the hull according to the local cascade $\left(\sigma_{t}, \prec_{t}, \rho_{t}\right)$. Since these neighbors come from the two disjoint sets $V\left(G_{t^{\prime}}\right) \backslash X_{t}$ and $V\left(G_{t^{\prime \prime}}\right) \backslash X_{t}$, say $\rho_{\text {int }}^{\prime}(u)$ from the first set and $\rho_{\text {int }}^{\prime \prime}(u)$ from the second set, it follows that $\pi_{t}\left(\sigma_{t}, \prec_{t}, \rho_{t}\right)$ can be chosen as the common extension to $V\left(G_{t}\right)$ of the two functions $\pi_{t^{\prime}}\left(\sigma_{t}, \prec_{t}, \rho_{t}+\rho_{\text {int }}^{\prime \prime}\right)$ and $\pi_{t^{\prime \prime}}\left(\sigma_{t}, \prec_{t}, \rho_{t}+\rho_{\text {int }}^{\prime}\right)$, where $\rho_{\text {int }}^{\prime}, \rho_{\text {int }}^{\prime \prime} \in[n-1]_{0}^{X_{t}}$ are chosen such that $\rho_{\text {int }}=\rho_{\text {int }}^{\prime}+\rho_{\text {int }}^{\prime \prime}$ and the cost of $\pi_{t}\left(\sigma_{t}, \prec_{t}, \rho_{t}\right)$ is minimized. Clearly, this can be done in time $n^{O(w)}$.

Since $T$ has order $O(w n)$, the overall computation takes $n^{O(w)}$ time, which completes the proof of Theorem 1.5.

\subsection{Partial incentives of interval graphs with bounded thresholds}

Our approach is similar to the one in [8] for computing a minimum dynamic monopoly of an interval graph with bounded thresholds. We first adapt an auxiliary result from [11,13] to the setting of partial 
incentives.

Lemma 2.3. Let $t$ be a non-negative integer. Let $G$ be a t-connected chordal graph, and let $\tau$ be a threshold function for $G$ with $\tau(u) \leq t$ for every vertex $u$ of $G$.

(i) For every clique $C$ in $G$ of order $t$ with vertices $v_{0}, v_{1}, \ldots, v_{t-1}$, the function

$$
\sigma(u)= \begin{cases}\tau\left(v_{i}\right)-i & , \text { if } u=v_{i} \text { for some } i \in[t-1]_{0}, \text { and } \\ 0 & , \text { otherwise }\end{cases}
$$

is a partial incentive of $(G, \tau)$.

(ii) $\operatorname{pi}(G, \tau) \leq\left(\begin{array}{c}t+1 \\ 2\end{array}\right)$.

Proof. (i) Clearly, $H=H_{(G, \tau-\sigma)}(\emptyset)$ contains $C$. If $G$ is a clique, the bound on $\tau$ implies that $H$ contains all vertices of $G$. If $G$ is not a clique, then $G$ has a simplicial vertex $u$ that does not belong to $C$. Since $G-u$ is $t$-connected, it follows, by an inductive argument, that $H$ contains $V(G) \backslash\{u\}$, and, since $d_{G}(u) \geq t, H$ contains also $u$, that is, $\sigma$ is a partial incentive of $(G, \tau)$.

(ii) This follows from the known fact that every $t$-connected chordal graph contains a clique of order $t$, and that $\sum_{i=0}^{t-1}(t-i)=\left(\begin{array}{c}t+1 \\ 2\end{array}\right)$.

Let $(G, \tau)$ be as in the statement of Theorem [1.6. We construct a sequence $G_{1} \subseteq G_{2} \subseteq \ldots \subseteq G_{k}$ of subgraphs of $G$ in such a way that $G_{k}=G$, and that Lemma 2.3 implies that the cost of every optimal partial incentive of $(G, \tau)$ within a suitable supergraph $\partial G_{i}$ of each graph $G_{i}-V\left(G_{i-1}\right)$ is at most $\left(\begin{array}{c}t+1 \\ 2\end{array}\right)$, cf. condition (iv) below. Clearly, we may assume that $G$ is connected. Let $n$ be the order of $G$. In linear time [10], we can determine an interval representation $(I(u))_{u \in V(G)}$ of $G$, that is, two distinct vertices $u$ and $v$ of $G$ are adjacent if and only if the intervals $I(u)$ and $I(v)$ intersect. By applying simple manipulations, we may assume that each interval $I(u)$ is closed, and that the $2 n$ endpoints of the $n$ intervals are all distinct.

Let $x_{1}<x_{2}<\ldots<x_{2 n}$ be the endpoints of the intervals. For each $i \in[2 n-1]$, let $C_{i}$ be the set of vertices $u$ of $G$ with $I_{i}:=\left[x_{i}, x_{i+1}\right] \subseteq I(u)$, and let $c_{i}=\left|C_{i}\right|$. Since each $x_{i}$ is either the right endpoint of exactly one interval or the left endpoint of exactly one interval, we have $\left|c_{i+1}-c_{i}\right|=1$ for every $i \in[2 n-1]$.

Lemma 2.4 (Bessy et al. [8]). If $C$ is a minimal vertex cut of $G$, then $C=C_{i}$ for some $i \in[2 n-2] \backslash\{1\}$ with $c_{i}<\min \left\{c_{i-1}, c_{i+1}\right\}$.

Let $j_{1}<j_{2}<\ldots<j_{k-1}$ be the indices $i$ in $[2 n-1] \backslash\{1\}$ with $c_{i}<\min \left\{c_{i-1}, c_{i+1}, t\right\}$, and let $j_{k}=2 n-1$. For $i \in[k]$, let $G_{i}$ be the subgraph of $G$ induced by $V_{i}:=C_{1} \cup \cdots \cup C_{j_{i}}$, and let $B_{i}=C_{j_{i}}$. Note that $B_{i}$ contains all vertices in $V_{i}$ that have a neighbor in $V(G) \backslash V_{i}$, and that $\left|B_{i}\right|<t$. Let $\partial V_{1}=V_{1}$, and, for $i \in[k] \backslash\{1\}$, let $\partial V_{i}=\left(V_{i} \backslash V_{i-1}\right) \cup B_{i-1}$. For $i \in[k]$, let $\partial G_{i}$ be the subgraph of $G$ induced by $\partial V_{i}$, cf Figure 1.

Lemma 2.5 (Bessy et al. [8]). For every $i \in[k]$, the graph $\partial G_{i}$ is either a clique of order at most $t$ or a $t$-connected graph. 

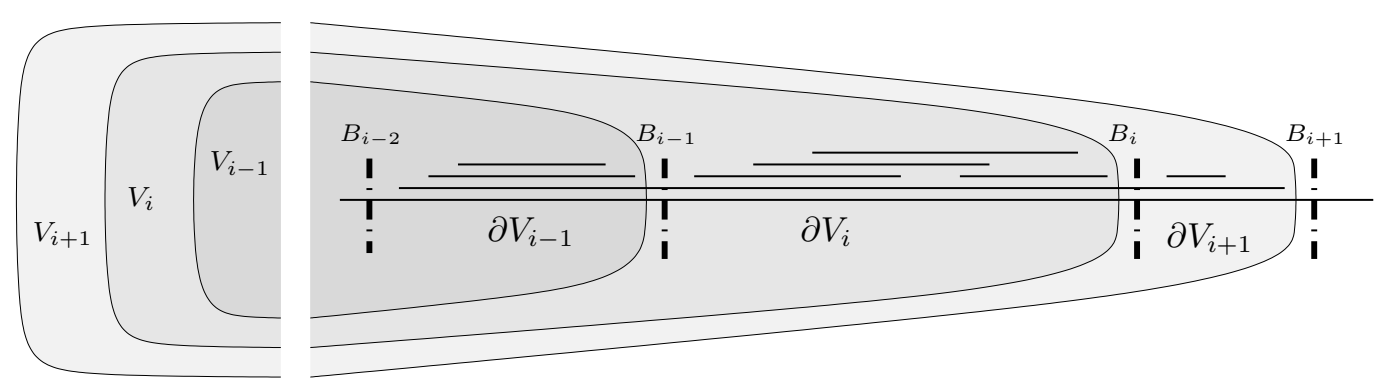

Figure 1: Sets $B_{i}, V_{i}$ and $\partial V_{i}$ on the interval representation of $G$ (for instance, $B_{i}$ contains all the intervals crossing the corresponding dotted line, $\partial V_{i}$ contains all the intervals intersecting the zone between $B_{i-1}$ and $B_{i}$, and $V_{i}$ contains all the intervals intersecting the corresponding zone).

Proof of Theorem 1.6. We use the same notation as above. Our approach is dynamic programming on the sequence $G_{1} \subseteq G_{2} \subseteq \ldots \subseteq G_{k}$. Similarly as for Theorem 1.5, we introduce the notion of a local cascade. More precisely, for every subgraph $G_{i}$, we consider

(i) all possible candidates for the restriction $\sigma_{i}$ of an optimal partial incentive to $B_{i}$,

(ii) all possible linear orders, in which the elements of $B_{i}$ enter the hull, and

(iii) all possible amounts of help that each vertex in $B_{i}$ receives from outside of $G_{i}$ when it enters the hull.

Formally, a local cascade for $G_{i}$ is a triple $\left(\sigma_{i}, \prec_{i}, \rho_{i}\right)$, where

(i) $\sigma_{i}: B_{i} \rightarrow[t]_{0}$

(ii) $\prec_{i}$ is a linear order on $B_{i}$ with $\left\{u \in B_{i}: \tau(u)-\sigma(u) \leq 0\right\} \prec_{i}\left\{v \in B_{i}: \tau(v)-\sigma(v)>0\right\}$,

(iii) $\rho_{i}: B_{i} \rightarrow[n-1]_{0}$.

Since $\left|B_{i}\right|<t$, there are $O\left((t+1)^{t-1}(t-1) ! n^{t-1}\right)=n^{O(t)}$ local cascades for $G_{i}$.

For a local cascade $\left(\sigma_{i}, \prec_{i}, \rho_{i}\right)$ for $G_{i}$, let $\pi_{i}\left(\sigma_{i}, \prec_{i}, \rho_{i}\right)$ be a function $\sigma \in[t]_{0}^{V\left(G_{i}\right)}$ with minimum cost such that $\left.\sigma\right|_{B_{i}}=\sigma_{i}$,

(iv) $\sum_{v \in \partial V_{j}} \sigma(v) \leq\left(\begin{array}{c}t+1 \\ 2\end{array}\right)$ for every $j \in[i]$, and

(v) there is a linear extension $\prec$ of $\prec_{i}$ to $G_{i}$ such that for every $u \in V\left(G_{i}\right)$

- either $u \in V\left(G_{i}\right) \backslash B_{i}$ and $\left|N_{G_{i}}^{\prec}(u)\right| \geq \tau(u)-\sigma(u)$,

- or $u \in B_{i}$ and $\left|N_{G_{i}}^{\prec}(u)\right| \geq \tau(u)-\sigma(u)-\rho_{i}(u)$,

where $N_{G_{i}}^{\prec}(u)$ denotes the neighbors of $u$ in $G_{i}$ that appear before $u$ in the linear order $\prec$. If no such function $\sigma$ exists, then we set all the values of $\pi_{i}\left(\sigma_{i}, \prec_{i}, \rho_{i}\right)$ to $\infty$. Otherwise, it is clear from the above definitions that $\pi_{i}\left(\sigma_{i}, \prec_{i}, \rho_{i}\right)$ is a partial incentive of $\left(G_{i}, \tau\right)$ which is optimal with respect to the conditions imposed by $\left(\sigma_{i}, \prec_{i}, \rho_{i}\right)$.

Note that $B_{k}$ consists of a single vertex, which does not have any neighbors outside of $G_{k}=G$. Therefore, using Lemma 2.3(ii) and Lemma 2.5, the following claim follow immediately from the definitions. 
Claim 2.4. The function $\pi_{k}\left(\sigma_{k}, \emptyset, 0\right)$ that minimizes the cost among all local cascades $\left(\sigma_{k}, \emptyset, 0\right)$ for $G_{k}$ is an optimal partial incentive of $(G, \tau)$.

The next two claims show how $\pi_{i}\left(\sigma_{i}, \prec_{i}, \rho_{i}\right)$ can be computed recursively.

Claim 2.5. For every local cascade $\left(\sigma_{1}, \prec_{1}, \rho_{1}\right)$ for $G_{1}, \pi_{1}\left(\sigma_{1}, \prec_{1}, \rho_{1}\right)$ can be computed in time $n^{O\left(t^{2}\right)}$.

Proof of Claim 2.5. Since $V_{1}=\partial V_{1}$, there are $O\left(n^{t(t+1) / 2}\right)$ candidates for a function $\sigma \in[t]_{0}^{V\left(G_{1}\right)}$ satisfying condition (iv). Hence, we can compute a function $\pi_{1}\left(\sigma_{1}, \prec 1, \rho_{1}\right)$ by brute-force in time $n^{O\left(t^{2}\right)}$.

Claim 2.6. For every $i \in[k] \backslash\{1\}$, and every local cascade $\left(\sigma_{i}, \prec_{i}, \rho_{i}\right)$ for $G_{i}$, given $\pi_{i-1}\left(\sigma_{i-1}, \prec_{i-1}\right.$ ,$\left.\rho_{i-1}\right)$ for all local cascades $\left(\sigma_{i-1}, \prec_{i-1}, \rho_{i-1}\right)$ for $G_{i-1}, \pi_{i}\left(\sigma_{i}, \prec_{i}, \rho_{i}\right)$ can be computed in time $n^{O\left(t^{2}\right)}$.

Proof of Claim [2.6. By definition, we have $B_{i} \cap V_{i-1} \subseteq B_{i-1}$. Therefore, the two sets $B_{i-1}^{\prime}=B_{i} \cap V_{i-1}$ and $B_{i-1}^{\prime \prime}=B_{i-1} \backslash B_{i-1}^{\prime}$ partition the set $B_{i-1}$. Note that $B_{i-1}^{\prime}=B_{i} \cap B_{i-1}$, and $B_{i-1}^{\prime \prime}=B_{i-1} \backslash B_{i}$, cf. Figure 2 ,

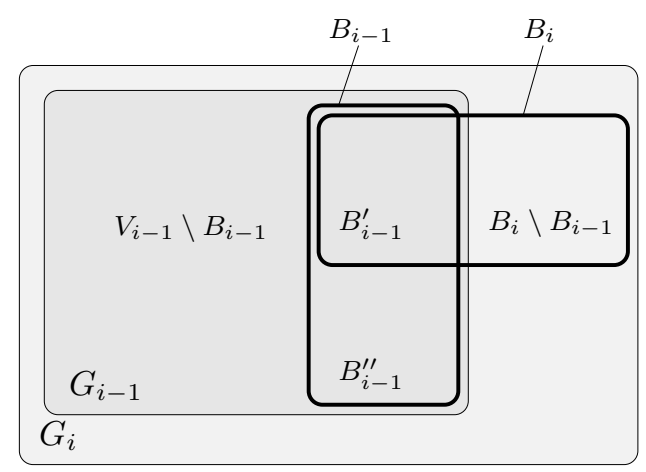

Figure 2: $G_{i}$ and relevant subsets of $V_{i}$.

Our approach to determine $\pi_{i}\left(\sigma_{i}, \prec_{i}, \rho_{i}\right)$ relies on considering all candidates $\sigma^{\prime \prime}$ for the restriction of a function $\sigma$ as in the definition of $\pi_{i}\left(\sigma_{i}, \prec_{i}, \rho_{i}\right)$ to the set $\partial V_{i} \backslash B_{i}$, that is, $\sigma^{\prime \prime}=\left.\sigma\right|_{\partial V_{i} \backslash B_{i}}$.

By (iv), this restriction satisfies $\sigma^{\prime \prime}\left(\partial V_{i} \backslash B_{i}\right) \leq\left(\begin{array}{c}t+1 \\ 2\end{array}\right)-\sigma_{i}\left(B_{i}\right)$.

In order to exploit the information encoded in the functions $\pi_{i-1}\left(\sigma_{i-1}, \prec_{i-1}, \rho_{i-1}\right)$, we decouple the formation of the hull in $G_{i}$ within the two graphs $\partial G_{i}$ and $G_{i-1}$. Accordingly, we consider all candidates for an extension $\prec_{(i-1, i)}$ of $\prec_{i}$ to $B_{i} \cup B_{i-1}$ specifying a possible order in which the vertices in $B_{i-1} \cup B_{i}$ enter the hull. By fixing the pairs $\left(\sigma^{\prime \prime}, \prec(i-1, i)\right.$, we specify that $\sigma_{i-1}=\left.\sigma_{i}\right|_{B_{i-1}^{\prime}}+\left.\sigma^{\prime \prime}\right|_{B_{i-1}^{\prime \prime}}$, and that the restriction of $\prec_{(i-1, i)}$ to $B_{i-1}$ is the linear order on $B_{i-1}$.

Formally, we consider all pairs $\left(\sigma^{\prime \prime}, \prec_{(i-1, i)}\right)$, where

- $\sigma^{\prime \prime} \in[t]_{0}^{\partial V_{i} \backslash B_{i}}$,

- $\sigma^{\prime \prime}\left(\partial V_{i} \backslash B_{i}\right) \leq\left(\begin{array}{c}t+1 \\ 2\end{array}\right)-\sigma_{i}\left(B_{i}\right)$

- $\prec_{(i-1, i)}$ is a linear extension of $\prec_{i}$ to $B_{i} \cup B_{i-1}$.

Let $\mathcal{S}$ denote the set of these pairs. Note that $|\mathcal{S}|=O\left(n^{t(t+1) / 2}(2 t-2) !\right)=n^{O\left(t^{2}\right)}$.

Let $\left(\sigma^{\prime \prime}, \prec_{(i-1, i)}\right)$ be in $\mathcal{S}$.

Assume that $v_{1} \prec_{(i-1, i)} \cdots \prec_{(i-1, i)} v_{p}$ is the linear order on $B_{i-1} \cup B_{i}$. 
For every $j \in[p]$ with $v_{j} \in B_{i-1} \cup B_{i}$, let $h_{j}$ be the number of neighbors of $v_{j}$ in the hull of the set $\left\{v_{\ell}: \ell \in[j-1]\right\}$ in

$$
\left(\partial G_{i}-\left\{v_{\ell}: \ell \in[p] \backslash[j-1]\right\}, \tau-\sigma_{i}-\sigma^{\prime \prime}\right) .
$$

If the hull of the set $B_{i-1} \cup B_{i}$ in $\left(\partial G_{i}, \tau-\sigma_{i}-\sigma^{\prime \prime}\right)$ does not equal $\partial V_{i}$ or if $h_{j}<\tau\left(v_{j}\right)-\sigma_{i}\left(v_{j}\right)-\rho_{i}\left(v_{j}\right)$ for some $j \in[p]$ with $v_{j} \in B_{i} \backslash B_{i-1}$, then we set all values of $s\left(\sigma^{\prime \prime}, \prec_{(i-1, i)}\right)$ to $\infty$. Note that these two cases correspond to violations of the condition (v). Hence, in what follows, we may assume that these two cases do not hold; in particular, $h_{j} \geq \tau\left(v_{j}\right)-\sigma_{i}\left(v_{j}\right)-\rho_{i}\left(v_{j}\right)$ for every $v_{j} \in B_{i} \backslash B_{i-1}$.

Let

- $\prec_{i-1}$ be the restriction of $\prec_{(i-1, i)}$ to $B_{i-1}$,

- $\rho_{i-1}\left(v_{j}\right)=\rho_{i}\left(v_{j}\right)+h_{j}$ for every $j \in[p]$ with $v_{j} \in B_{i-1}^{\prime}$, and

- $\rho_{i-1}\left(v_{j}\right)=h_{j}$ for every $j \in[p]$ with $v_{j} \in B_{i-1}^{\prime \prime}$.

Let

$$
s\left(\sigma^{\prime \prime}, \prec_{(i-1, i)}\right)=\left.\sigma_{i}\right|_{B_{i} \backslash B_{i-1}}+\left.\sigma^{\prime \prime}\right|_{\partial V_{i} \backslash\left(B_{i-1} \cup B_{i}\right)}+\pi_{i-1}\left(\left.\sigma_{i}\right|_{B_{i-1}^{\prime}}+\left.\sigma^{\prime \prime}\right|_{B_{i-1}^{\prime \prime}}, \prec_{i-1}, \rho_{i-1}\right) .
$$

Note that also in this case some values of $s\left(\sigma^{\prime \prime}, \prec_{(i-1, i)}\right)$ can be $\infty$. Note, furthermore, that, for every $v_{j} \in B_{i-1}^{\prime}$, the value of $\rho_{i-1}\left(v_{j}\right)$ has a contributing term $\rho_{i}\left(v_{j}\right)$ quantifying the help from outside of $V_{i}$ as well as a contributing term $h_{j}$ quantifying the help from outside of $V_{i-1}$ but from inside of $V_{i}$. For every $v_{j} \in B_{i-1}^{\prime \prime}$, there is no help from outside of $V_{i}$, that is, the first term disappears. In view of the above explanation, it now follows easily that the function $s\left(\sigma^{\prime \prime}, \prec_{(i-1, i)}\right)$ of minimum cost with $\left(\sigma^{\prime \prime}, \prec_{(i-1, i)}\right)$ in $\mathcal{S}$ is a suitable choice for $\pi_{i}\left(\sigma_{i}, \prec_{i}, \rho_{i}\right)$. Since $\mathcal{S}$ has $n^{O\left(t^{2}\right)}$ elements, and each function $s\left(\sigma^{\prime \prime}, \prec_{(i-1, i)}\right)$ can be determined in $n^{O(1)}$ time, the claim follows.

Since $k \leq n$, and there are only $n^{O(t)}$ local cascades for each $G_{i}$, the Claims 2.4 2.6 complete the proof of Theorem 1.6.

\subsection{Approximation algorithms for planar graphs}

First, we use Baker's [5] layering technique for constructing a PTAS for maximum degenerate sets in planar graphs.

Proof of Theorem 1.7. Let $\epsilon$ and $(G, \kappa)$ be as in the statement. We fix a plane embedding of $G$. Let $L(0), L(1), \ldots, L(\ell)$ be the layers of $G$, that is,

- $L(0)$ is the set of vertices on the outer face of $G$,

- $L(i)$ is the set of vertices on the outer face of $G-(L(0) \cup \ldots \cup L(i-1))$ for $i \geq 1$, and

- $V(G)=\bigcup_{i=0}^{\ell} L(i)$.

Let $k=\left\lceil\frac{1}{\epsilon}\right\rceil$. For every $i \in[k-1]_{0}$, the components of the graph $G-X(i)$, where

$$
X(i)=\bigcup_{j=0}^{\left\lfloor\frac{\ell-i}{k}\right\rfloor} L(i+j k),
$$


are the subgraphs $G(i, j)$ of $G$ induced by

$$
L(i+(j-1) k+1) \cup L(i+(j-1) k+2) \cup \ldots \cup L(i+j k-1)
$$

for $j \in\left[\left\lfloor\frac{\ell-i-1}{k}\right\rfloor+1\right]_{0}$, where we set $L(j):=\emptyset$ for $j<0$ or $j>\ell$. Since each $G(i, j)$ is induced by at most $k$ consecutive layers, the treewidth of these graphs, and, hence, also of $G-X(i)$ is at most $3 k-1$ [9]. By the main result of Ben-Zwi et al. [7], and by the duality of dynamic monopolies and degenerate sets, we can determine in time $n^{O(k)}$ maximum $\left.\kappa\right|_{V(G(i, j))}$-degenerate sets $I(i, j)$ in $G(i, j)$ for all $i \in[k-1]_{0}$ and all $j \in\left[\left\lfloor\frac{\ell-i-1}{k}\right\rfloor+1\right]_{0}$. Let

$$
I(i)=\bigcup_{j=0}^{\left\lfloor\frac{\ell-i-1}{k}\right\rfloor+1} I(i, j)
$$

and let $I$ be the largest of the sets $I(0), \ldots, I(k-1)$. Clearly, $I$ is a $\kappa$-degenerate set in $G$.

Let $I_{\max }$ be a maximum $\kappa$-degenerate set in $G$. Since $\left|I_{\max }\right|=\sum_{i=0}^{k-1}\left|I_{\max } \cap X(i)\right|$, there is an index $i^{*} \in[k-1]_{0}$ with $\left|I_{\max } \cap X\left(i^{*}\right)\right| \leq \frac{1}{k}\left|I_{\max }\right| \leq \epsilon\left|I_{\max }\right|$. Since $I_{\max } \cap V(G(i, j))$ is a $\left.\kappa\right|_{V(G(i, j)) \text {-degenerate }}$ set in $G_{i, j}$, we obtain

$$
\begin{aligned}
|I| \geq\left|I\left(i^{*}\right)\right| & =\sum_{j=0}^{\left\lfloor\frac{\ell-i^{*}-1}{k}\right\rfloor+1}\left|I\left(i^{*}, j\right)\right| \\
& \geq \sum_{j=0}^{\left\lfloor\frac{\ell-i^{*}-1}{k}\right\rfloor+1}\left|I_{\max } \cap V\left(G\left(i^{*}, j\right)\right)\right| \\
& =\left|I_{\max }\right|-\left|I_{\max } \cap X\left(i^{*}\right)\right| \\
& \geq(1-\epsilon)\left|I_{\max }\right|,
\end{aligned}
$$

which completes the proof.

For the proof of Theorem 1.8, we adapt the approach of [1].

For a graph $G$ and a subset $A$ of vertices of $G$, let $N^{+}(A)=\bigcup_{v \in A} N_{G}(v) \backslash A$ be the outside neighbors of $A$, and let $N^{-}(A)=N^{+}(V(G) \backslash A)$ be the boundary of $A$. For a threshold function $\tau$ for $G$ and two sets of vertices $A$ and $B$ of $G$, we call $B$ an $A$-strong region if $B \nsubseteq H_{(G, \tau)}\left(A \cup N^{+}(B)\right)$. Otherwise we call $B$ an $A$-weak region.

The following lemma is a straightforward generalization of Lemmas 3.3-3.5 in [1], and can be proved analogously.

Lemma 2.6. Let $G$ be a graph with threshold function $\tau$ and let $A$ and $B$ be sets of vertices of $G$.

(i) $B$ is an $A$-strong region if and only if $B \cap(D \backslash A) \neq \emptyset$ for every dynamic monopoly $D$ of $(G, \tau)$ containing A.

(ii) If $B$ is an $A$-weak region with $N^{-}(B) \subseteq A$, then $B \subseteq H_{(G, \tau)}(A)$.

(iii) If $B$ is an $A$-strong region and $Y$ is a subset of $B$ with $Y \subseteq H_{(G, \tau)}(A)$ and $N^{-}(Y) \subseteq A$, then $B \backslash Y$ is an A-strong region. 
Proof of Theorem 1.8 . Let $(G, \tau)$ be as in the statement and let $\left(T,\left(X_{i}\right)_{i \in V(T)}\right)$ be a rooted treedecomposition of $G$ of width $w$. For simplicity, we may assume that $V(T)=[k]$ and that $1, \ldots, k$ is a reversed BFS-ordering of the nodes of $T$, which is rooted at $k$. As in the definition of a treedecomposition in subsection 2.1, for a node $i$ of $T$, let $G_{i}$ be the subgraph of $G$ induced by the bags of the node $i$ and all its descendants in $T$. We describe how to compute a dynamic monopoly for $(G, \tau)$ in time $O\left(n^{2} m\right)$ with approximation guarantee $w+1$. Let $A_{0}=\emptyset, A_{1}, \ldots, A_{k}$ be sets of vertices of $G$, recursively defined for every $i \in[k]$, such that

$$
A_{i}= \begin{cases}A_{i-1} \cup X_{i} & , \text { if } V\left(G_{i}\right) \text { is an } A_{i-1} \text {-strong region, and } \\ A_{i-1} & , \text { if } V\left(G_{i}\right) \text { is an } A_{i-1} \text {-weak region. }\end{cases}
$$

By the definition of a tree-decomposition, the boundary vertices of $G_{i}$ lie in the bag $X_{i}$, that is, $N^{-}\left(V\left(G_{i}\right)\right) \subseteq X_{i}$, and for every child node $j$ of $i$, the boundary vertices of $G_{j}$ lie in $X_{i} \cap X_{j}$, that is, $N^{-}\left(V\left(G_{j}\right)\right) \subseteq X_{i} \cap X_{j}$, and thus $N^{-}\left(V\left(G_{j}\right)\right) \subseteq X_{i}$.

First, we show that the set $A_{k}$ is a dynamic monopoly for $(G, \tau)$.

Claim. Let $i \in[k]$. If $V\left(G_{i}\right)$ is an $A_{i-1}$-strong region, then $V\left(G_{i}\right) \subseteq H_{(G, \tau)}\left(A_{i-1} \cup X_{i}\right)$.

Proof of the Claim. We prove the claim by induction on the height of the subtree $T_{i}$. The statement is true if $i$ is a leaf of $T$ since $V\left(G_{i}\right)=X_{i}$. Hence, let $i_{1}, \ldots, i_{\ell}$ be the children of $i$ in $T$. For every $j \in[\ell]$, when the algorithm examined $V\left(G_{i_{j}}\right)$ either $V\left(G_{i_{j}}\right)$ was $A_{i_{j}-1}$-weak or $A_{i_{j}-1}$-strong. If $V\left(G_{i_{j}}\right)$ was $A_{i_{j}-1}$-weak, by Lemma 2.6 (ii), we have

$$
V\left(G_{i_{j}}\right) \subseteq H_{(G, \tau)}\left(A_{i_{j}-1} \cup N^{-}\left(V\left(G_{i_{j}}\right)\right)\right) \subseteq H_{(G, \tau)}\left(A_{i_{j}-1} \cup X_{i}\right) \subseteq H_{(G, \tau)}\left(A_{i} \cup X_{i}\right) .
$$

If $V\left(G_{i_{j}}\right)$ was $A_{i_{j}-1}$-strong, by induction, we have

$$
V\left(G_{i_{j}}\right) \subseteq H_{(G, \tau)}\left(A_{i_{j}-1} \cup X_{i_{j}}\right) \subseteq H_{(G, \tau)}\left(A_{i}\right)
$$

Altogether, since $V\left(G_{i}\right)=X_{i} \cup \bigcup_{j=1}^{\ell} V\left(G_{i_{j}}\right)$, this proves the claim.

We obtain that $A_{k}$ is a dynamic monopoly for $(G, \tau)$, because $V\left(G_{k}\right)=V(G)$ and

- either $V\left(G_{k}\right)$ is an $A_{k-1}$-strong region, thus, $A_{k}=A_{k-1} \cup X_{k}$ and by the above claim $V\left(G_{k}\right) \subseteq$ $H_{(G, \tau)}\left(A_{k-1} \cup X_{k}\right)$,

- or $V\left(G_{k}\right)$ is an $A_{k-1}$-weak region, thus, $A_{k}=A_{k-1}$ and again by Lemma 2.6 (ii), $V\left(G_{k}\right) \subseteq$ $H_{(G, \tau)}\left(A_{k-1} \cup N^{-}\left(V\left(G_{k}\right)\right)\right)=H_{(G, \tau)}\left(A_{k}\right)$ since $N^{-}\left(V\left(G_{k}\right)\right)=\emptyset$.

Let $i_{1} \leq i_{2} \leq \ldots \leq i_{b}$ be all the nodes of $T$ that each corresponds to an $A_{i_{j}-1}$-strong region. For

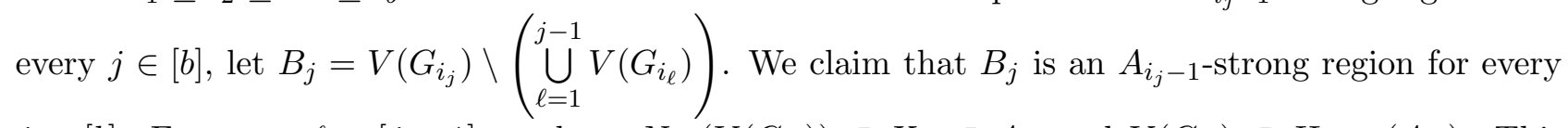
$j \in[b]$. For every $\ell \in[j-1]$, we have $N^{-}\left(V\left(G_{i_{\ell}}\right)\right) \subseteq X_{i_{\ell}} \subseteq A_{i_{j}}$ and $V\left(G_{i_{\ell}}\right) \subseteq H_{(G, \tau)}\left(A_{i_{j}}\right)$. This implies that $N^{-}\left(\bigcup_{\ell=1}^{j-1} V\left(G_{i_{\ell}}\right)\right) \subseteq A_{i_{j}}$ and $\bigcup_{\ell=1}^{j-1} V\left(G_{i_{\ell}}\right) \subseteq H_{(G, \tau)}\left(A_{i_{j}}\right)$. Hence, by Lemma 2.6 (iii), $B_{j}$ is 
$A_{i_{j}-1}$-strong. By construction, the sets $B_{1}, \ldots, B_{b}$ are pairwise disjoint, and for each such set, a bag $X_{i_{j}}$ is added to $A_{i_{j}-1}$. This implies that

$$
A_{k} \leq(w+1) b \leq(w+1) \operatorname{dyn}(G, \tau)
$$

since each bag has size at most $w+1$ and $\operatorname{dyn}(G, \tau) \geq b$, by Lemma 2.6 (i). This proves the approximation guarantee.

Finally, we show that the described algorithm can be implemented with running time $O\left(n^{2} m\right)$. Recall that the given tree-decomposition has $O(n)$ nodes. For each node $i$, it can be checked whether $V\left(G_{i}\right)$ is an $A_{i-1}$-strong region by constructing the hull $H_{(G, \tau)}\left(A_{i-1} \cup N^{+}\left(V\left(G_{i}\right)\right)\right)$ in time $O(n m)$. This completes the proof.

Corollary 1.9 follows easily from Theorem 1.8 and the fact that for a planar graph, a treedecomposition of width $O(\sqrt{n})$ can be found in time $O\left(n^{3 / 2}\right)[3]$.

In fact, in [17] it was shown that if every subgraph of a graph $G$ with order $n$ has a balanced separator of size at most $k$ then $G$ has treewidth at most $105 k$, where a balanced separator $S$ is a set of vertices of $G$ such that every component of $G-S$ has size at most $2 n / 3$. The balanced separation number is the smallest integer $k$ fulfilling this condition. Hence, for every graph of order $n$ and balanced separation number at most $O\left(n^{1-\epsilon}\right)$, Theorem 1.8 yields an approximation algorithm with ratio $O\left(n^{1-\epsilon}\right)$, given the corresponding tree-decomposition. Note that the result of Lipton and Tarjan [22] immediately implies a balanced separation number of size $O(\sqrt{n})$ for planar graphs.

\section{References}

[1] A. Aazami, K. Stilp, Approximation algorithms and hardness for domination with propagation, SIAM Journal on Discrete Mathematics 23.3 (2009) 1382-1399.

[2] E. Ackerman, O. Ben-Zwi, G. Wolfovitz, Combinatorial model and bounds for target set selection, Theoretical Computer Science 411 (2010) 4017-4022.

[3] J. Alber, H.L. Bodlaender, H. Fernau, T. Kloks, R. Niedermeier, Fixed parameter algorithms for dominating set and related problems on planar graphs, Algorithmica 33 (2002) 461-493.

[4] S. Arnborg, D.G. Corneil, A. Proskurowski, Complexity of finding embeddings in a $k$-tree, SIAM Journal on Algebraic and Discrete Methods 8 (1987) 277-284.

[5] B.S. Baker, Approximation algorithms for NP-complete problems on planar graphs, Journal of the ACM 41 (1994) 153-180.

[6] R. Barbosa, D. Rautenbach, V. Fernandes dos Santos, J.L. Szwarcfiter, On minimal and minimum hull sets, Electronic Notes in Discrete Mathematics 44 (2013) 207-212.

[7] O. Ben-Zwi, D. Hermelin, D. Lokshtanov, I. Newman, Treewidth governs the complexity of target set selection, Discrete Optimization 8 (2011) 87-96.

[8] S. Bessy, S. Ehard, L.D. Penso, D. Rautenbach, Dynamic monopolies for interval graphs with bounded thresholds, arXiv:1802.03935. 
[9] H.L. Bodlaender, A partial k-arboretum of graphs with bounded treewidth, Theoretical Computer Science 209 (1998) 1-45.

[10] K.S. Booth, G.S. Lueker, Testing for the consecutive ones property, interval graphs, and graph planarity using PQ-tree algorithms, Journal of Computer and System Sciences 13 (1976) 335-379.

[11] C.C. Centeno, M.C. Dourado, L.D. Penso, D. Rautenbach, J.L. Szwarcfiter, Irreversible conversion of graphs, Theoretical Computer Science 412 (2011) 3693-3700.

[12] N. Chen, On the approximability of influence in social networks, SIAM Journal on Discrete Mathematics 23 (2009) 1400-1415.

[13] C.-Y. Chiang, L.-H. Huang, B.-J. Li. J. Wu, H.-G. Yeh, Some results on the target set selection problem, Journal of Combinatorial Optimization 25 (2013) 702-715.

[14] G. Cordasco, L. Gargano, A. Rescigno, U. Vaccaro, Optimizing spread of influence in social networks via partial incentives, Lecture Notes in Computer Science 9439 (2015) 119-134.

[15] E.D. Demaine and M. Hajiaghayi, The bidimensionality theory and its algorithmic applications, Computer Journal 51 (2008) 292-302.

[16] P.A. Dreyer Jr., F.S. Roberts, Irreversible k-threshold processes: Graph-theoretical threshold models of the spread of disease and of opinion, Discrete Applied Mathematics 157 (2009) 16151627 .

[17] Z. Dvorák, S. Norin, Treewidth of graphs with balanced separations, arXiv:1408.3869.

[18] M.R. Garey, D.S. Johnson, L. Stockmeyer, Some simplified NP-complete graph problems, Theoretical Computer Science 1 (1976) 237-267.

[19] D. Kempe, J. Kleinberg, E. Tardos, Maximizing the spread of influence through a social network, Theory of Computing 11 (2015) 105-147.

[20] T. Kloks, Treewidth. Computations and approximations, Lecture Notes in Computer Science 842 (1994).

[21] J. Kynčl, B. Lidický, T. Vyskočil, Irreversible 2-conversion set in graphs of bounded degree, Discrete Mathematics and Theoretical Computer Science 19 (2017) \# 3.

[22] R.J. Lipton, R.E. Tarjan, A separator theorem for planar graphs, SIAM Journal on Applied Mathematics 36 (1979) 177-189.

[23] D. Reichman, New bounds for contagious sets, Discrete Mathematics 312 (2012) 1812-1814. 